\title{
HPV genotype analysis for women in Shaanxi Province of China
}

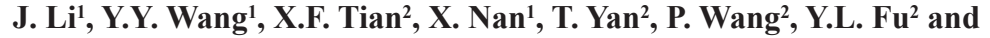 \\ G.Q. Wang ${ }^{2}$
}

${ }^{1}$ Tumor Research Department, Shaanxi Provincial Tumor Hospital, Xi'an, China ${ }^{2}$ Gynecologic Oncology Center, Shaanxi Provincial Tumor Hospital, Xi'an, China

Corresponding author: J. Li

E-mail: wonder_689@126.com

Genet. Mol. Res. 15 (4): gmr15047178

Received January 5, 2016

Accepted August 22, 2016

Published November 3, 2016

DOI http://dx.doi.org/10.4238/gmr15047178

Copyright (C) 2016 The Authors. This is an open-access article distributed under the terms of the Creative Commons Attribution ShareAlike (CC BY-SA) 4.0 License.

\begin{abstract}
The aim of this study was to examine the subtype distribution of human papilloma virus (HPV) in women in the Shaanxi Province of China. A DNA chip, along with polymerase chain reaction amplification and reverse dot blot technology, was adopted to analyze the HPV genotypes of 22,937 cases of cervical cell specimens. The HPV infection rate was $18.70 \%$, wherein high-risk, low-risk, and high- and low-risk multiple infection rates were 15.75, 2.96 and $1.91 \%$, respectively. High-risk infections accounted for $84.20 \%$ of total infections. The rate of HPV infection in women with rural residence, high school education or less, a low income, or age over 40 years was significantly higher than that in the control group (negative HPV infection women). Of the 18 detected high-risk HPV subtypes, the most common in single infections were, in the order of prevalence, HPV16, 58, 18, 52, 33, and 56. For multiple high-risk infections, the most common subtypes in the order of prevalence were HPV16, 52, $58,18,56$, and 33. Age was a factor in the rate of infection, as the 41-
\end{abstract}

Genetics and Molecular Research 15 (4): gmr15047178 
50-year age group had a significantly higher risk of infection than the other groups $(\mathrm{P}<0.05)$. In multiple infections, double infections were common, accounting for $77.10 \%$ of multiple infections, and triple or more infections were more common in women aged 51-60 years. In Shaanxi Province, high-risk HPV infection in women was mainly attributed to rural residence, age over 40 years, low income, and low education level.

Key words: Age distribution; Human papilloma virus; Subtype distribution; Multiple infection; Cervical cancer

\section{INTRODUCTION}

Cervical cancer is one of the most common malignancies in women worldwide and its incidence ranks second only to breast cancer in China. In recent years, its high occurrence has been primarily found in women aged 45-49 years. The occurrence and development of cervical cancer and cervical human papillomavirus (HPV) infection are closely related. Based on the pathogenic differences, HPV can be divided into high-risk and low-risk categories (Muñoz et al., 2003). The low-risk genotypes do not cause malignant lesions, resulting only in genital warts and low-grade cervical intraepithelial neoplasia grade 1 (CIN1) (Woodman et al., 2001), while the high-risk genotypes are a risk factor for cervical cancer (Sandri et al., 2009). Studies have shown that $99.7 \%$ of cancer patients have high-risk HPV infection (Bory et al., 2002). Worldwide, HPV subtypes are significantly different between countries, regions and ethnicities; therefore, understanding the predominant genotypes and epidemiological characteristics of HPV infections in each population can provide guidance for the prevention of cervical cancer in that particular region.

\section{MATERIAL AND METHODS}

\section{Subjects}

\section{General information}

The cervical squamous epithelium cell specimens were collected from female patients who were enrolled in the Shaanxi Provincial Tumor Hospital for treatment, opportunistic screening, health screening, and women's "two cancer" (breast and cervical cancers) screening from January 2011 to May 2014. Selection epidemiologic factors (age, region, education, and average annual income) of cervical lesions were investigated.

\section{Inclusion criteria}

The subjects met the following requirements: resident of a city or county in Shaanxi province, sound intelligence, not pregnant, aged 18 to 65 years, married or with more than 1 year of sexual history, no cervical colonization or history of hysterectomy, no systemic infection or autoimmune diseases, no history of oral immune suppressants, and no vaginal drug or sexual activity 3 days before the gynecological exam. Cervical cancer patients had not undergone surgery, radiotherapy, or chemotherapy.

Genetics and Molecular Research 15 (4): gmr15047178 


\section{Age distribution}

There were a total of 22,937 cases of women aged 19 to 65 years (mean age, $42.38 \pm$ 20.17 years). From this, 76 women were younger than 20 years, 1033 women were 21 to 30 years old, 4788 women were 31 to 40 years old, 9679 women were 41 to 50 years old, 5298 women were 51 to 60 years old, and 2063 women were older than 60 years.

\section{Reagents}

The HPV genotyping assay kit was provided by Shenzhen Asia Bio Technology Co., Ltd., (China) which simultaneously detects 18 high-risk subtypes $(16,18,31,33,35,39,45$, $51,52,53,56,58,59,66,68,73,82$, and 83$)$ and 5 low-risk subtypes $(6,11,42,43$, and 81$)$ of HPV. PALL nylon membranes with an aperture of $0.45 \mathrm{~mm}$ and a thickness of $6.0 \pm 0.5 \mathrm{mils}$ were purchased from the Pall Corporation (New Port Richey, FL, USA).

\section{Methods}

\section{Specimen collection and storage}

Colposcopy or vaginal dilator was used to fully expose the cervix, which was wiped with a cotton swab to take a sample of secretions. A cervical brush was then placed in the cervix, spun clockwise 4-5 times and slowly withdrawn to obtain a sufficient sample of cervical epithelial cells. The brush handle was broken off and the brush head was placed into the cervix elution tube, which contained cell preservation solution. The sample was stored at $-20^{\circ} \mathrm{C}$.

\section{DNA extraction}

The cervical brush was thoroughly rinsed and the eluent was transferred into a $1.5-\mathrm{mL}$ tube, which was centrifuged at 13,000 $\mathrm{g}$ for $10 \mathrm{~min}$, and the supernatant was discarded. The cells were lysed with $50 \mu \mathrm{L}$ buffer, added to multiple wells, heated at $100^{\circ} \mathrm{C}$ for $10 \mathrm{~min}$, and immediately centrifuged at $13,000 \mathrm{rpm}$ for $10 \mathrm{~min}$. The middle layer of supernatant contained the DNA solution.

\section{Polymerase chain reaction (PCR) amplification}

PCR tubes with $20 \mu \mathrm{L}$ reaction mixtures were centrifuged at $5000 \mathrm{rpm}$ for $2 \mathrm{~s}$, and then 2 $\mu \mathrm{L}$ mineral oil was added with either $5 \mu \mathrm{L}$ extracted DNA, blank control (added water), or positive control (HPV positive), resulting in a final reaction volume of $27 \mu \mathrm{L}$. Amplification conditions were as follows: $50^{\circ} \mathrm{C}$ for $15 \mathrm{~min}, 95^{\circ} \mathrm{C}$ for $10 \mathrm{~min}$, and then 35 cycles of $94^{\circ} \mathrm{C}$ for $30 \mathrm{~s}, 42^{\circ} \mathrm{C}$ for $90 \mathrm{~s}$, and $72^{\circ} \mathrm{C}$ for $30 \mathrm{~s}$. A final extension step at $72^{\circ} \mathrm{C}$ for $5 \mathrm{~min}$ completed the reaction.

\section{Hybridization, incubation, and blotting}

The samples, film strip (multiple film strips with 23 HPV probes), and all PCR products were added to $5-6 \mathrm{~mL}$ solution A ( $2 \mathrm{X}$ saline-sodium citrate, $0.1 \% \mathrm{SDS}$ ) in a $15-\mathrm{mL}$ tube. After denaturation in a boiling water bath for $10 \mathrm{~min}$, the tube was immediately placed

Genetics and Molecular Research 15 (4): gmr15047178 
in a $51^{\circ} \mathrm{C}$ hybridization chamber for $1.5 \mathrm{~h}$. Then the film strips were transferred into $40 \mathrm{~mL}$ preheated solution B $(0.5 \mathrm{X}$ saline-sodium citrate, $0.1 \% \mathrm{SDS}, \mathrm{pH} 7.4)$ and incubated at $51^{\circ} \mathrm{C}$ for $5 \mathrm{~min}$. The film strips were transferred to POD incubation solution and incubated at room temperature for $30 \mathrm{~min}$. The incubation solution was discarded and the pellet was washed twice for 5 min with solution $\mathrm{A}$ at room temperature and solution $\mathrm{C}(0.1 \mathrm{M}$ sodium citrate) for $2 \mathrm{~min}$. The film strips were placed in freshly prepared colored liquid $(19 \mathrm{~mL}$ solution $\mathrm{C}, 1 \mathrm{~mL}$ TMB, $10 \mu \mathrm{L} \mathrm{30 \%} \mathrm{H}_{2} \mathrm{O}_{2}$ ) to develop for $15 \mathrm{~min}$ in the dark. Last, the film strips were soaked in water and stored in a sealed bag at $4^{\circ} \mathrm{C}$. Blue spots were regarded as positive and the presence of particular genotypes was determined by a special analyzer. The positive control was used to monitor the accuracy and reliability of the system.

\section{Statistical analysis}

Data were analyzed using Student $t$-test or nonparametric tests, are reported as means \pm standard deviation, and rates were compared with the chi-square test. Multifactor logistic regression analysis was used to analyze the high-risk factors for HPV infection. All analysis used the statistical software SPSS 16.0 (Chicago, IL, USA), with $\mathrm{P}<0.05$ regarded as statistically significant.

\section{RESULTS}

\section{Demographic characteristics and high-risk HPV infection status}

From the 22,937 cervical cell specimens to be genotyped for HPV infection, the average age of the subjects was $41.38 \pm 20.17$ years; of these subjects, 9408 lived in urban areas and 13,529 lived in rural areas. The general demographic characteristics and rate of high-risk HPV infections are shown in Table 1. A total of 3612 cases had a high-risk infection (including a single high-risk as well as high- and low-risk multiple infections) and the total rate of high-risk infection was $15.75 \%$. Univariate analysis showed that the high-risk HPV infection rate was significantly higher in rural women with high school or lesser education, low-income women, and women over 40 years of age, as compared to the control group $(\mathrm{P}<0.05)$.

Table 1. Demographic characteristics and high-risk HPV infection status in women.

\begin{tabular}{|c|c|c|c|c|c|}
\hline \multicolumn{2}{|l|}{ Demographics } & \multirow{2}{*}{$\begin{array}{c}\text { Case }(\mathrm{N}) \\
5,991\end{array}$} & \multirow{2}{*}{$\begin{array}{c}\text { Ratio (\%) } \\
26.12\end{array}$} & \multirow{2}{*}{$\begin{array}{c}\text { High-risk HPV infections }(\%) \\
714(11.92)\end{array}$} & \multirow{2}{*}{$\begin{array}{c}\mathrm{P} \\
0.000\end{array}$} \\
\hline Age & $\leq 40$ years & & & & \\
\hline & $>40$ years & 16,946 & 73.88 & $2,898(17.10)$ & \\
\hline \multirow[t]{2}{*}{ Region } & City & 9,408 & 41.02 & $951(10.11)$ & 0.000 \\
\hline & Rural & 13,529 & 58.98 & $2,661(19.67)$ & \\
\hline \multirow[t]{3}{*}{ Education } & Primary school/illiterate & 3,393 & 14.79 & $634(18.69)$ & 0.006 \\
\hline & Middle school & 11,819 & 51.53 & $1,961(16.59)$ & 0.003 \\
\hline & College & 7,725 & 33.68 & $1,017(13.17)$ & \\
\hline \multirow[t]{2}{*}{ Average annual income } & $<1,538$ (US\$) & 14,228 & 62.03 & $2,543(17.87)$ & 0.000 \\
\hline & $\geq 1,538$ (US\$) & 8,709 & 37.97 & $1,069(12.27)$ & \\
\hline
\end{tabular}

\section{Overall status of HPV infection in all age groups}

Women aged 41 to 50 years had the highest HPV infection rate $(\mathrm{P}<0.05$; Figure 1$)$, with total and high-risk infection rates of $21.93 \%(2232 / 10,177)$ and $18.64 \%(1897 / 10,177)$,

Genetics and Molecular Research 15 (4): gmr15047178 
respectively. The second highest prevalence of HPV infection was the age group of 51 to 60 years, with total and high-risk infection rates of $18.52 \%(1010 / 5453)$ and $16.12 \%(879 / 5453)$, respectively. Women under the age of 20 had the lowest total and high-risk infection rates $[5.41 \%(4 / 74)$ and $4.05 \%(3 / 74)$, respectively].

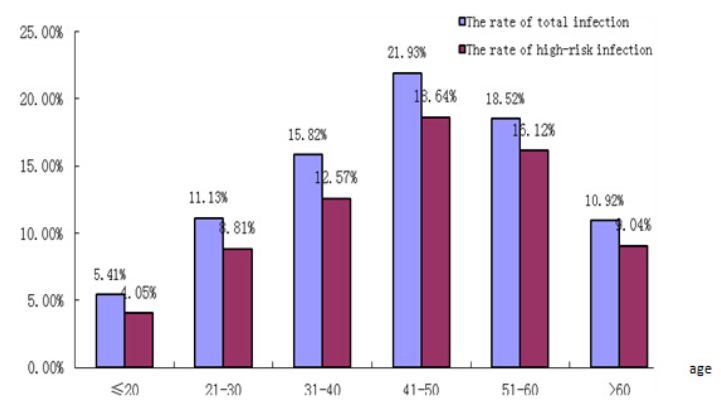

Figure 1. Rate of total and high-risk HPV infection in women in all age groups.

\section{Distribution of HPV genotypes}

All 18 high-risk HPV genotypes were detected (Table 2), with HPV16 being the most common one. HPV16 accounted for $40.69 \%$ of total single high-risk infection genotypes (1058/2600), followed by HPV58 (9.81\%), HPV81 (8.42\%), HPV18 (7.08\%), HPV52 (6.96\%), and HPV43 (5.88\%). In multiple infections, the HPV16 subtype was also the most common [16.99\% (325/1913)], followed by HPV52 (12.60\%), HPV58 (9.57), HPV81 (9.25\%), HPV18 (7.95\%), and HPV42 (7.58\%). The overall detection rate of the HPV16 subtype was $24.39 \%$, which was significantly higher than any other subtype $(\mathrm{P}<0.001)$. Other than HPV16, 58, $52,81,18$, and 42 subtypes were the next most commonly identified genotypes in order of prevalence. HPV16 accounted for 30.64\% of high-risk multiple infections (1383/4513), followed by HPV58, 52, 18, 56, and 33, which together accounted for $68.47 \%(3090 / 4513)$ of high-risk multiple infections. Out of the five low-risk genotypes, HPV81 was the most common and accounted for $34.23 \%(396 / 1157)$ of total HPV infections, and it was far more prevalent than the other low-risk subtypes $(\mathrm{P}<0.001)$. HPV42 and HPV43 accounted for $82.54 \%(955 / 1157)$ of total HPV infections.

\section{Distribution of low-risk and high-risk HPV infection in all age groups}

In all examined subjects, the rate of single low-risk infection (including single low-risk infection and low-risk subtypes in multiple infections) was 2.96\% (678/22,937), accounting for $15.80 \%$ (678/4290) of all HPV infections. The prevalence of high-risk subtypes (including single high-risk infection and high-risk subtypes in multiple infection) was as high as $15.75 \%$ (3612/22,937), accounting for $84.20 \%$ of all HPV infections (3612/4290). The infection rate of high-risk subtypes was significantly higher than that of low-risk subtypes $(\mathrm{P}<0.000)$. The rate of high-risk HPV infection in the 41-50 year age group was the highest at $18.64 \%$ $(1897 / 10,177)$, which was significantly higher than that of any other age group $(\mathrm{P}<0.05)$. The group of subjects under 20 years of age had only three cases (4.05\%) of high-risk infection and 1 case $(1.35 \%)$ of low-risk infection. The group of subjects over 60 years of age also had low 
rates of high- and low-risk infections $(9.04 \%$ (96/1062) and 1.88\% (20/1062), respectively). These data are summarized in Table 3.

\begin{tabular}{|c|c|c|c|c|c|}
\hline \multicolumn{2}{|l|}{ Subtype } & Single infection & Multiple infections & Total & Rate \\
\hline \multirow[t]{18}{*}{ High-risk } & 16 & 1058 & 325 & 1383 & 24.39 \\
\hline & 18 & 184 & 152 & 336 & 5.93 \\
\hline & 31 & 73 & 99 & 172 & 3.03 \\
\hline & 33 & 128 & 120 & 248 & 4.37 \\
\hline & 35 & 68 & 79 & 147 & 2.59 \\
\hline & 39 & 46 & 42 & 88 & 1.55 \\
\hline & 45 & 30 & 39 & 69 & 1.22 \\
\hline & 51 & 58 & 85 & 143 & 2.52 \\
\hline & 52 & 181 & 241 & 422 & 7.44 \\
\hline & 53 & 93 & 112 & 205 & 3.62 \\
\hline & 56 & 119 & 144 & 263 & 4.64 \\
\hline & 58 & 255 & 183 & 438 & 7.73 \\
\hline & 59 & 80 & 46 & 126 & 2.22 \\
\hline & 66 & 83 & 73 & 156 & 2.75 \\
\hline & 68 & 88 & 116 & 204 & 3.60 \\
\hline & 73 & 22 & 31 & 53 & 0.94 \\
\hline & 82 & 3 & 7 & 10 & 0.18 \\
\hline & 83 & 31 & 19 & 50 & 0.88 \\
\hline Hish-risk total & & 2600 & 1913 & 4513 & 79.60 \\
\hline \multirow{5}{*}{ Low-risk } & 6 & 56 & 59 & 115 & 2.03 \\
\hline & 11 & 59 & 28 & 87 & 1.53 \\
\hline & 42 & 142 & 145 & 287 & 5.06 \\
\hline & 43 & 153 & 119 & 272 & 4.80 \\
\hline & 81 & 219 & 177 & 396 & 6.98 \\
\hline Low-risk total & & 629 & 528 & 1157 & 20.40 \\
\hline Overall total & & 3229 & 2441 & 5670 & 100.00 \\
\hline
\end{tabular}

Each subtype in the multiple infection was counted separately.

Table 3. Distribution of low-risk and high-risk HPV infections in all age groups.

\begin{tabular}{|c|c|c|c|c|c|c|}
\hline & & & Age & ear) & & \\
\hline & $\leq 20$ & $21-30$ & $31-40$ & $41-50$ & $51-60$ & $>60$ \\
\hline Total case & 74 & 1033 & 5138 & 10177 & 5453 & 1062 \\
\hline Low-risk infection* & 1 & 24 & 167 & 335 & 131 & 20 \\
\hline High-risk infection** & 3 & 91 & 646 & 1897 & 879 & 96 \\
\hline
\end{tabular}

*Including low-risk single and multiple infections. **Including high-risk single and multiple infections.

\section{Single and multiple HPV infection status in different age groups}

Of all HPV infections, single HPV infection was most common, with an infection rate of $14.08 \%(3229 / 22,937)$. This accounted for $75.27 \%$ of HPV infections $(3229 / 4290)$, which was significantly higher than the rate of double infection $(1061 / 22,937)$, which was $4.63 \%$ and accounted for $19.07 \%$ of HPV infections $(818 / 4290, \mathrm{P}<0.001)$. Single low- and high-risk infection rates were $2.74 \%(629 / 22,937)$ and $11.34 \%(2600 / 22,937)$, respectively, accounting for $14.66 \%$ (629/4290) and 60.61\% (2600/4290) of total HPV infections, respectively $(\mathrm{P}<0.001)$. The rate of double infection was $3.57 \%(818 / 22,937)$, accounting for $19.07 \%$ of all HPV infections (818/4290). There were only 49 cases with double infection of low-risk subtypes, while the double high-risk infection and highand low-risk multiple infection rates were $2.02 \%(464 / 22,937)$ and $1.33 \%(305 / 22,937)$,

Genetics and Molecular Research 15 (4): gmr15047178 
respectively, accounting for $10.82 \%(464 / 4290)$ and $7.11 \%(305 / 4290)$ of all HPV infections, respectively. The rate of triple infection was $0.87 \%(200 / 22,937)$, accounting for $4.66 \%$ of HPV infection (200/4290). The quadruple infection rate was only $0.19 \%$ $(43 / 22,937)$, accounting for $1.00 \%$ of HPV infections (43/4290). In all groups, the single HPV subtype infection rate in the $41-50$ age group was $16.64 \%(1693 / 10,177)$. This accounted for $39.46 \%$ of all HPV infections (1693/4290), which was significantly higher than all other age groups $(\mathrm{P}<0.05)$. These data are summarized in Table 4.

Table 4. Single and multiple HPV infection status in different age groups.

\begin{tabular}{|c|c|c|c|c|c|c|c|}
\hline & & & & & & & Total \\
\hline & $\leq 20$ & $21-30$ & $31-40$ & $41-50$ & $51-60$ & $>60$ & \\
\hline Single low-risk & 1 & 21 & 157 & 316 & 114 & 20 & 629 \\
\hline Single high-risk & 3 & 64 & 495 & 1377 & 584 & 77 & 2600 \\
\hline Double low-risk* & 0 & 3 & 10 & 19 & 17 & 0 & 49 \\
\hline Double high-risk & 0 & 9 & 93 & 252 & 101 & 9 & 464 \\
\hline Double multiple & 0 & 9 & 26 & 167 & 96 & 7 & 305 \\
\hline Triple high-risk & 0 & 3 & 12 & 45 & 26 & 2 & 88 \\
\hline Triple multiple & 0 & 6 & 14 & 44 & 47 & 1 & 112 \\
\hline Quadruple high-risk & 0 & 0 & 2 & 3 & 6 & 0 & 11 \\
\hline Quadruple multiple & 0 & 0 & 2 & 1 & 7 & 0 & 10 \\
\hline Five high-risk & 0 & 0 & 1 & 2 & 4 & 0 & 7 \\
\hline Five multiple & 0 & 0 & 1 & 2 & 3 & 0 & 6 \\
\hline Six high-risk & 0 & 0 & 0 & 1 & 1 & 0 & 2 \\
\hline Six multiple & 0 & 0 & 0 & 2 & 3 & 0 & 5 \\
\hline Seven high-risk & 0 & 0 & 0 & 1 & 0 & 0 & 1 \\
\hline Seven multiple & 0 & 0 & 0 & 0 & 1 & 0 & 1 \\
\hline Total & 4 & 115 & 813 & 2232 & 1010 & 116 & 4290 \\
\hline
\end{tabular}

*No low-risk was found in low-risk or more multiple infections.

\section{DISCUSSION}

HPV is a member of the Papovaviridae A virus family and is a papilloma vacuole virus. It has closed circular double-stranded DNA with about 8000 base pairs and 3 gene domains (E, L, and UCR). The E domain encodes early proteins, such as E1, E2, E4, E5, $\mathrm{E} 6$, and E7, which are mainly related to the biological functions of viral genome replication, transcription regulation, and induction of transformed host cells. The L region encodes L1 and L2 proteins, which are important for viral packaging. The UCR contains the origin of replication and regulatory elements for HPV genomic DNA (Doorbar, 2006). HPV is present in most mammals and birds. Currently there are more than 200 HPV subtypes known, of which more than 30 cause human disease. Different subtypes of HPV can lead to various diseases; the high-risk infections can cause cervical, anal or penile cancer and the low-risk infections cause external genital warts and low-grade cervical intraepithelial lesions (Clifford et al., 2003). An active sex life and poor immune status of underprivileged adult women predispose to HPV infection, and 70 to $80 \%$ of women will have at least one HPV infection in their lifetime. The majority of these infections occur in women over the age of 35 , when the infection rate may reach $20-50 \%$. Despite the high rate of infection, HPV is usually cleared by the immune system within $8-10$ months and only $10-15 \%$ of patients will continue to be infected, which could potentially lead to cervical cancer. In China, there is a high rate of HPV infection, with over 18 million new cases each year (Kong and Qu, 2009), and the incidence continues to increase annually. Therefore, timely HPV screening and appropriate early intervention for

Genetics and Molecular Research 15 (4): gmr15047178 
cervical lesions is particularly important for cervical cancer prevention and treatment.

In this study, 22,937 women from Shaanxi Province received HPV infection genotyping, in which the total number of infections was 4290 and the total infection rate was $18.70 \%$ $(4290 / 22,937)$. The number of high-risk infections was 3612 , with an infection rate of $15.75 \%$ $(3612 / 22,937)$ and accounting for $84.20 \%$ of the total infections $(3612 / 4290)$. This result is consistent with previous reports (Wang et al., 2010; Yang et al., 2012). Statistical analysis showed that high-risk HPV infection in women over 40 years of age from rural areas, with low income and low education level was higher (Table 1). In addition, reports of HPV infection in women from different age groups vary in different parts of China. This study showed that the lowest rates of total and high-risk infection were in the under 20 years of age group (5.41 and $4.05 \%$, respectively), while the $41-50$ years age group had the highest rates (21.93 and $18.64 \%$, respectively). This was followed by the 51-60 years age group (total rate was $18.52 \%$ and high-risk rate was $16.12 \%$ ). This is consistent with the high incidence of cervical cancer in the Chinese population, but different from some domestic reports (Li et al., 2010; Dai and Zhang, 2013). It can be speculated that younger women have lesser sexual experience and stronger immune function, thus quickly removing the HPV viral infection, which results in a transient infection. From the 41-50 year age group, many women experienced stress, had an unhealthy lifestyle, relatively frequent sexual activity, and reduced immune function. This hindered immune function could enable the virus to persist, increasing the potentiality of highrisk HPV infection and the susceptibility to long-term infections.

In North America, high-risk HPV infection includes the subtypes HPV16, 53, 52, 18, and 39. In Europe, the common subtypes are HPV16, 18, 31, 33, and 58, and in Africa, the common subtypes are HPV16, 52, 18, 58, and 31 (de Sanjosé et al., 2007). In Tianjin, China, the common high-risk HPV subtypes are HPV16, 58, 52, 33, and 56. In Nanjing, China, the common subtypes are HPV16, 58, 33, 18, and 31, and in Chongqing, China, the common subtypes are HPV16, 58, 52, 18, and 31. Last, in Gansu, China, the common subtypes are HPV16, 58, 52, 31, and 18 (Wang et al., 2010; Yang et al., 2012; Zhang et al., 2013). This study detected 23 subtypes of HPV, from which 18 were high-risk $(16,18,31,33,35,39,45$, $51,52,53,56,58,59,66,68,73,82$, and 83) in women in Shaanxi Province, China. In single HPV subtype infection cases, the most common subtypes were HPV16, 58, 81, 18, 52, and 43 , accounting for $47.79 \%$ of HPV infections (2050/4290). The most common single highrisk subtypes were HPV16, 58, 52, 18, 56, and 33, which together accounted for $68.47 \%$ of infections (3090/4513). In multiple HPV infections, the most common subtypes were HPV16, $52,58,81,18$, and 42 , accounting for $50.10 \%$ (1223/2441) of all HPV infections. HPV16 had an overall detection rate of $24.39 \%$, which is significantly higher than that of other HPV subtypes $(\mathrm{P}<0.001)$. Distribution of low-risk subtypes in HPV infection in all age groups was consistent with the distribution of high-risk HPV infections (Table 3). We detected five low-risk subtypes of HPV, and the most common single low-risk subtypes were HPV81, 43, 42,11 , and 6 , accounting for $14.66 \%$ of HPV infections (629/4290). The most common lowrisk subtypes in multiple infections were HPV81, 42, 43, 6, and 11, accounting for $6.98 \%$ of multiple infections (396/5670). Low-risk HPV infections in China are mostly reported to be caused by HPV6 and 11 (Lee et al., 2003; Wang et al., 2010). In Lee's study, HPV81 was the most common low-risk subtype in single $(34.82 \%, 219 / 629)$ and multiple infections $(33.52 \%$, 177/528), which has also been reported in Chongqing, China (Kong and Qu, 2009; Wang et al., 2010). The most prevalent subtypes in low-risk single infections were HPV81, 43, 42, 11, and 6, and the most prevalent subtypes in low-risk multiple infections were HPV81, 42, 43, 6,

Genetics and Molecular Research 15 (4): gmr15047178 
and 11 (Table 2). The results reported in this study do show some discrepancies from reports in other areas of China (Wang et al., 2010; Jiang et al., 2012).

This study showed that HPV infection has a clear regional character, meaning that different countries and regions have their own unique subtype distribution characteristics. Numerous studies have demonstrated the epidemiological and molecular biology of HPV and have shown that HPV infection can be prevented by a vaccine, which can also greatly reduce or even eliminate cervical cancer. HPV infection has obvious geographical features, which creates pressure to make a vaccine that targets all prominent subtypes, and targeted research and development for a vaccine with region-specific subtypes will result in lower levels of cervical cancer. Therefore, knowledge regarding the distribution of HPV subtypes in a given region has great value for screening, prevention, treatment, and vaccine development for cervical cancer.

Single HPV infection is about three times more common than multiple infections (Jiang et al., 2012). In Jiang et al. (2012)'s study, a single HPV infection accounted for $75.27 \%$ (3229/4290) of the total infections, and the HPV16 subtype was the most common $(24.66 \%$, $1058 / 4290)$. The rate of multiple infection was $24.73 \%$ (1061/4290), resulting in a ratio of four single infections to one multiple infection, which is slightly higher than that in previous reports (Jiang et al., 2012) (Table 3). In multiple infections, the most common combination was HPV16 with other subtypes $(13.31 \%, 325 / 2441)$, followed by HPV52, 58, 81, 18, and 42. Multiple infections accounted for $40.23 \%$ (982/2441) of all infections (Table 2). Studies have shown that the risk of cervical cancer can be increased nearly 20 times with a single HPV infection and 32 times with multiple HPV infections. Compared to single infection, multiple HPV infection results in a higher viral load, leading to a higher risk of cancer, faster lesion development, and higher recurrence (Lee et al., 2003; Fan et al., 2009; Zhao et al., 2009). It is suggested that, when focusing on single clinical infections, regular follow-up and monitoring of multiple HPV infections should be performed to reduce the incidence of cervical cancer.

In different age groups, the highest risk of infection was noted in the 31-50 years group, followed by the 51-60 years and 21-30 years groups, with age £20 years and age $>60$ years having the lowest risk $(\mathrm{P}<0.05)$. The risk of infection was the highest in the $41-50$ years age group, followed by the 51-60 years $(16.12 \%)$ and the $31-40$ years age groups $(12.57 \%)$. The risk of infection was lowest in the $£ 20$ yearsage group (4.05\%) (Table 3$)$. The infection rate for single low-risk infection was $14.66 \%$ (629/4290), and single high-risk infections accounted for $60.61 \%(2600 / 4290)$ of total infections. The majority of these infections were double high-risk and double multiple infections $(94.01 \%, 769 / 818)$. For low-risk multiple infections, there were only double low-risk subtype infections and infection with three or more subtypes was not observed (Table 4). For infection with four or more multiple subtypes, there were only 43 cases, accounting for $1.00 \%$ of all infections (43/4290) and $1.76 \%$ of multiple infections (43/2441). The rate of high- and low-risk multiple infections was $1.91 \%$ $(439 / 22,937)$, accounting for $10.23 \%$ of total multiple infections (439/4290) (Table 4).

In different age groups, a single infection was still the most common form of infection, followed by superinfection. For a single HPV infection, the infection rate in the 4150 years age group was $16.64 \%(1693 / 10,177)$ and high-risk single infection rate was $13.53 \%$ $(1377 / 10,177)$, which was significantly higher than that in the other groups $(\mathrm{P}<0.05)$. In women who were 31-60 years old, the double infection rate was similar. In women under20 years of age, there was only one case of single infection and no multiple infections. In the 2130 years and $>60$ years age groups, one case with four or more subtypes was found. Multiple

Genetics and Molecular Research 15 (4): gmr15047178 
infections with six or seven subtypes only occurred in the 41-60 years age group.

The analysis for infection with three or more subtypes showed that the 51-60 year age group had the highest rate at 1.79\% (98/5453), accounting for $9.70 \%$ of total infections $(98 / 1010)$, which was significantly higher than that for the other age groups $(\mathrm{P}<0.05)$. This result shows that the prevalence of multiple infection increases with age. After 60 years of age, a significant reduction was found in multiple infections (Table 4). The women over 40 years of age had a high incidence of cervical cancer. It takes about 5-15 years to progress from HPV infection to cervical cancer; therefore, women who are 30-60 years old should be primary subjects for HPV screening for reducing the incidence of cervical cancer.

In Europe and other developed countries, the HPV test has been regarded as one of the preferred screening methods for prevention of cervical cancer because it has much higher sensitivity than cytology, making it important for primary screening, detection, and treatment (Garcia-Echeverria and Sellers, 2008). As a result, the incidence and mortality of cervical cancer in Europe and America have significantly reduced after the effective implementation of screening. In China, similar measures should be taken in cervical cancer screening for women to enhance cancer health education, such as explanation of risk factors and early signs of cervical cancer. Better screening methods for HPV need to be developed in order to detect lesions before the occurrence of cervical cancer, thus achieving early diagnosis and treatment. In addition, development of region-specific HPV vaccines based on epidemiological data will effectively reduce the incidence of cervical cancer.

\section{Conflicts of interest}

The authors declare no conflict of interest.

\section{ACKNOWLEDGMENTS}

We thank all the patients who were enrolled to this study.

\section{REFERENCES}

Bory JP, Cucherousset J, Lorenzato M, Gabriel R, et al. (2002). Recurrent human papillomavirus infection detected with the hybrid capture II assay selects women with normal cervical smears at risk for developing high grade cervical lesions: a longitudinal study of 3,091 women. Int. J. Cancer 102: 519-525.http://dx.doi.org/10.1002/ijc.10735

Clifford GM, Smith JS, Plummer M, Muñoz N, et al. (2003). Human papillomavirus types in invasive cervical cancer worldwide: a meta-analysis. Br. J. Cancer 88: 63-73. http://dx.doi.org/10.1038/sj.bjc.6600688

Dai HY and Zhang XJ (2013). Study on subtype age distribution and multiple infection of human papillomavirus (HPV) in Yongchuan area of Chongqion. Chongqing Med. 42: 619-621.

de Sanjosé S, Diaz M, Castellsagué X, Clifford G, et al. (2007). Worldwide prevalence and genotype distribution of cervical human papillomavirus DNA in women with normal cytology: a meta-analysis. Lancet Infect. Dis. 7: 453459. http://dx.doi.org/10.1016/S1473-3099(07)70158-5

Doorbar J (2006). Molecular biology of human papillomavirus infection and cervical cancer. Clin. Sci. 110: 525-541. http://dx.doi.org/10.1042/CS20050369

Fan WS, Li YL, Yang YZ, Sun Z, et al. (2009). Clinical analysis of human papillomavirus in paraffin-embedded cervical lesion tissues by gene chip technology. Chin. J. Nosocomiol. 19: 745-747.

Garcia-Echeverria C and Sellers WR (2008). Drug discovery approaches targeting the PI3K/Akt pathway in cancer. Oncogene 27: 5511-5526. http://dx.doi.org/10.1038/onc.2008.246

Jiang D, Ye JD, Zhang XP, Xu A, et al. (2012). Analysis of infection of among 10000 female migrant population in Shenzhen. Chin. J. of PHM 28: 796-798.

Genetics and Molecular Research 15 (4): gmr15047178 
Kong DX and Qu QX (2009). Expression and their correlation of COX-2 and VEGF-C in cervical cancer. J. Int. Obstet. Gynecol 36: 151-153.

Lee SA, Kang D, Seo SS, Jeong JK, et al. (2003). Multiple HPV infection in cervical cancer screened by HPVDNAChip. Cancer Lett. 198: 187-192. http://dx.doi.org/10.1016/S0304-3835(03)00312-4

Li C, Wu M, Wang J, Zhang S, et al. (2010). A population-based study on the risks of cervical lesion and human papillomavirus infection among women in Beijing, People's Republic of China. Cancer Epidemiol. Biomarkers Prev. 19: 2655-2664.http://dx.doi.org/10.1158/1055-9965.EPI-10-0212

Muñoz N, Bosch FX, de Sanjosé S, Herrero R, et al.; International Agency for Research on Cancer Multicenter Cervical Cancer Study Group (2003). Epidemiologic classification of human papillomavirus types associated with cervical cancer. N. Engl. J. Med. 348: 518-527. http://dx.doi.org/10.1056/NEJMoa021641

Sandri MT, Riggio D, Salvatici M, Passerini R, et al. (2009). Typing of human papillomavirus in women with cervical lesions: prevalence and distribution of different genotypes. J. Med. Virol. 81: 271-277. http://dx.doi.org/10.1002/ jmv.21382

Wang Y, Wu ZZ and Zhou QY (2010). Epidemiological characteristics of cervical human papillomavirus women in Gansu. Matern. Child Health Care China 25: 5371-5373.

Woodman CB, Collins S, Winter H, Bailey A, et al. (2001). Natural history of cervical human papillomavirus infection in young women: a longitudinal cohort study. Lancet 357: 1831-1836. http://dx.doi.org/10.1016/S0140-6736(00)04956-4

Yang J, Zhou DP, Chen FX, Peng JH, et al. (2012). Investigation and analysis on HPV infection status of 2497 patients with gynecological disease in Chongqing. J. Chongqing Med. Univ. 37: 347-349.

Zhang JS, Geng JX, Han CR, Liu X, et al. (2013). Study on human papillomavirus genetic profile in 3678 cervical cell samples of married women. Int. Lab. Med. 34: 439-441.

Zhao R, Zhang WY, Wu MH, Zhang SW, et al. (2009). Human papillomavirus infection in Beijing, People's Republic of China: a population-based study. Br. J. Cancer 101: 1635-1640.http://dx.doi.org/10.1038/sj.bjc.6605351

Genetics and Molecular Research 15 (4): gmr15047178 